\title{
Investigating Adipokine levels in the sera of patients with myocardial infarction in a 6-month follow up
}

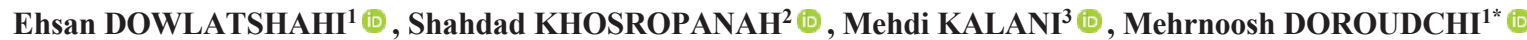

${ }^{1}$ Department of Immunology, School of Medicine, Shiraz University of Medical Sciences, Shiraz, Iran

${ }^{2}$ Department of Cardiology, School of Medicine, Shiraz University of Medical Sciences, Shiraz, Iran

${ }^{3}$ Department of Immunology, Professor Alborzi Clinical Microbiology Research Center, Nemazee Hospital, Shiraz

University of Medical Sciences, Shiraz, Iran

\begin{abstract}
\begin{tabular}{lllll}
\hline Received: 16.02 .2021 & $\bullet$ & Accepted/Published Online: 25.03 .2021 & Final Version: 30.08 .2021
\end{tabular}
\section{Abstract}

Adipokines are peptides that regulate endothelial function, inflammation, blood pressure, and hemostasis. We measured Leptin, Adiponectin, Resistin and Adipsin in a cohort of 36 STEMI patients. Serum levels of Adipokines were measured in three time-points by a multiplex assay. A significant difference in Adipsin concentration between day-5 (T5) and day-180 (T180) post-MI was observed. Resistin levels decreased significantly from day 0 (T0) to T5 and T180. Adiponectin/Resistin ratio increased from T0 to T180. Leptin at T0 and T5 were higher in nonsmokers. Adipsin at T0 was inversely correlated with heart rate, respiratory rate and pulse rate. Adiponectin/Resistin ratio at T180 negatively correlated with respiratory-rate. Adiponectin/Resistin ratio at T180 was higher in patients with grade-1 atrioventricular (AV) block. Anteroseptal hypokinesia (AH) correlated with Resistin at T0 and Adipsin at T5 while Leptin at T0 and T5 correlated with AH. Adiponectin/Resistin ratio at T180 was; however, lower in patients with AH. A decreasing trend in Resistin and its T0 association with AH plus correlation of Leptin at T0 and T5 with AH show the effect of Adipokines on mechanical complications after MI. We suggest that Adipokine networks have both beneficial and harmful effects and may be new cardiac biomarkers and/or drug targets.
\end{abstract}

Keywords: adipokine, biomarker, inflammation, myocardial infarction

\section{Introduction}

The rate of cardiovascular diseases in middle- and lowincome countries is growing rapidly (1). One of the major cardiovascular pathologies occurs in myocardial infarction (MI) which is the most detrimental atherosclerosis related complication (2). Different risk factors such as stress, hypertension, physical inactivity, obesity, diabetes mellitus and cigarette smoking lead to ischemia, reperfusion injury and MI (3). A complex cascade of inflammatory events results in progression of atherosclerosis, plaque rupture, and emboli in MI. Lymphocyte infiltration is found in MI patients who die immediately, in short (four weeks), or long (four months) time after coronary thrombosis (2).

Adipose tissue, consisted of adipocytes, fibroblasts, lymphocytes, macrophages and other cells, is an active tissue which expresses a number of biologically active molecules named Adipokines (4). It is now well received that obesity, insulin resistance, type 2 diabetes, high blood pressure and cardiovascular (CV) system are highly influenced by the action of Adipokines (5-8). Adipokines are peptides that are produced by Adipocytes, endothelial and immune cells, fibroblasts, and other cells which regulate endothelial function, inflammation, blood pressure, hemostasis, adipogenesis, immune cell migration, adipocyte metabolism and function $(9,4,10)$.
Leptin was the first discovered Adipokine in 1994. Administration of Leptin has cardio-protective effects e.g., reduces the extent of myocardial infarction (MI) and protects against reperfusion damage (11). Nevertheless, most studies consider Leptin as a hazardous Adipokine in cardiovascular system that is associated with atherosclerosis, hypertension and the metabolic syndrome. Also, Leptin affects blood pressure, insulin resistance, platelet aggregation and has proinflammatory effects (11). Higher levels of Leptin are associated with myocardial infarction (MI) and stroke, independent of traditional risk factors or obesity $(11,12)$.

Resistin, another Adipokine, is a $12.5-\mathrm{kDa}$ cysteine-rich polypeptide, and a member of the FIZZ (found in inflammatory zones) family of proteins (13). There is a relationship between Resistin and classic mediators of inflammation, such as interleukin-6 (IL-6) and tumor necrosis factor alpha (TNF- $\alpha$ ) (14). In fact, Resistin has been shown to have pleiotropic functions in metabolism and physiology with roles in inflammation (15), endothelial dysfunction (16) cardiomyocyte function (17), and cholesterol metabolism (18). In vitro experiments have shown that Resistin activates endothelial cells to upregulate the expression of adhesion molecules and inflammatory cytokines, induces proliferation and migration of smooth muscle cells, and accelerates 
transformation of foam cells (19). Therefore, Resistin may contribute to the atherosclerotic process by stimulating multiple pro-atherosclerotic pathways (20). Increased serum Resistin levels are shown to be associated with coronary artery disease (CAD) and the risk of cardiovascular death (21).

Adiponectin, also named as ACRP30 and AdipoQ, is an adipose tissue-derived Adipokine $(21,22)$. Many Adipokines are positively regulated by adiposity, but adiponectin levels in plasma are negatively regulated by accumulation of body fat (23). Clinical studies implicate hypo-adiponectinemia in the pathogenesis of CAD. Adiponectin plays a protective role in the development of insulin resistance, hypertension and cardiovascular disease (CVD) (24).

Another Adipokine, Adipsin, is mainly expressed in adipocytes and is involved in the activation of the alternative pathway of complement with the acyl-stimulating protein as the final component. The positive correlation between Adipsin levels and body mass suggests the role of Adipsin in the increase of fat mass through acyl-stimulating protein synthesis, increase of differentiation of pre-adipocytes, and synthesis of triglycerides (25). Adipsin level is reduced in murine models of obesity but it either increases or remains unchanged in obese human subjects (26). Despite a large body of data on many Adipokines, the information on the Adipsin and its correlation with MI is scarce. In this study, we measured the serum levels of Leptin, Resistin, Adiponectin, and Adipsin in a cohort of patients with MI in a 6-month follow up and investigated their association with the risk factors and clinical criteria of patients.

\section{Materials and methods}

\subsection{Study population}

In this study, a total of 50 individuals with STEMI who developed a first coronary event were recruited. All patients with chest pain complaints, increase in TnT levels and ST elevation in anterolateral and anteroseptal leads, if aged $<75$ years, were eligible for inclusion in the study. Myocardial infarction was diagnosed based on the presenting electrocardiogram (ECG) in combination with serial TnT measurements. Selective coronary angiography in the hospital course was used to further confirm the diagnosis. Patients with Chronic Renal Failure (CRF), autoimmune diseases, and cardiogenic shock were excluded from the study. All patients were monitored for six months. After six months echocardiography was done for all the patients for evaluation of LV systolic function. LV ejection fraction was assessed by echocardiography performed by a single blinded expert operator. Follow-up end points were defined as a new ACS (e.g. cardiac ischemia and AMI) or a repeat coronary revascularization (PCI and $\mathrm{CABG}$ ) after the initial event, which were combined as nonfatal events. The fatal events comprised all cases of all-cause mortality. Otherwise, followup ended at the date of withdrawal from the study or at six months after entry.

For each patient, demographic and clinical information including age, gender, and history of hypertension (Systolic blood pressure $>150 \mathrm{mmHg}$ and Diastolic blood pressure $>$ $90 \mathrm{mmHg}$ ), hyperlypidemia (Total Cholesterol $>240 \mathrm{mg} / \mathrm{dL}$ or LDL- Cholesterol $>160 \mathrm{mg} / \mathrm{dL}$ or Triglycerides $>200$ $\mathrm{mg} / \mathrm{dL}$ ), diabetes mellitus, obesity and BMI, and smoking were obtained and recorded. This datasheet as well as their laboratory data were used for statistical analysis. A resting heart rate between 60 and 100 beats per minute was considered normal. Higher than 100 beats per minute (tachycardia) and lower than 60 beats per minute (bradycardia) were considered not normal. Respiratory rate was recorded as the number of breaths taken per minute. The resting respiration rate of 12 to 20 breaths per minute was considered normal. A respiration rate under 12 or over 25 breaths per minute while resting was considered not normal. The sera were collected at three time points as: 1) On admission (T0) 2) $5^{\text {th }}$ day of hospitalization (T5) 3) 180 days after MI (T180). Of 50 original individuals who consented to enter the study only 36 remained available for the last (T180) sampling. Therefore, the levels of Adipokines were measured in the sera of 36 STEMI patients (24 men and 12 women) in the T0 and T5. In T180, three patients had been expired and therefore, the number of cases decreased to 33 at this time point. All demographic, general and clinical information of the patients were recorded at the time of sampling by collaborating cardiologist.

\subsection{Serum separation}

Blood samples were drawn from patients and aliquots of serum were collected from each individual at the three time points. The sera were frozen in $-40^{\circ} \mathrm{C}$ until tested.

\subsection{Adipokine multiplex assay}

Circulating levels of serum Adiponectin, Resistin, Adipsin, Leptin were determined in three time-points with a commercially available multiplex bioassay (LEGENDplex ${ }^{\mathrm{TM}}$ Human Metabolic Panel 1 (4-plex)). The assay was performed according to the manufacturer's specifications. The samples were tested using a FACS Calibur Flow Cytometer (BD) instrument system.

\subsection{Serum separation}

Blood samples were drawn from patients and aliquots of serum were collected from each individual at the three time points. The sera were frozen in $-40^{\circ} \mathrm{C}$ until tested.

\subsection{Adipokine multiplex assay}

Circulating levels of serum Adiponectin, Resistin, Adipsin, Leptin were determined in three time-points with a commercially available multiplex bioassay (LEGENDplex ${ }^{\mathrm{TM}}$ Human Metabolic Panel 1 (4-plex)). The assay was performed according to the manufacturer's specifications. The samples were tested using a FACS Calibur Flow Cytometer (BD) instrument system. 
Table 1. Adipokine levels based on demographical features of AMI patients in each time point

\begin{tabular}{|c|c|c|c|c|c|c|c|c|c|c|c|c|c|c|}
\hline \multirow{2}{*}{\multicolumn{2}{|c|}{ Subjects }} & \multirow[t]{2}{*}{ No (\%) } & \multicolumn{3}{|c|}{$\begin{array}{c}\text { Adiponectin } \\
\text { Mean }(\mathrm{SD}) \\
\left(\mathrm{ng} / \mathrm{ml} \times \mathbf{1 0}^{\mathbf{3}}\right) \\
\end{array}$} & \multicolumn{3}{|c|}{$\begin{array}{c}\text { Adipsin } \\
\text { Mean (SD) } \\
\left(\mathrm{pg} / \mathrm{ml} \times 10^{3}\right)\end{array}$} & \multicolumn{3}{|c|}{$\begin{array}{c}\text { Resistin } \\
\text { Mean (SD) } \\
\left(\mathrm{pg} / \mathrm{ml} \times 10^{3}\right) \\
\end{array}$} & \multicolumn{3}{|c|}{$\begin{array}{c}\text { Leptin } \\
\text { Mean (SD) } \\
\left(\mathrm{pg} / \mathrm{ml} \times 10^{3}\right)\end{array}$} \\
\hline & & & T0 & T5 & T180 & T0 & T5 & T180 & T0 & T5 & T180 & T0 & T5 & T180 \\
\hline No. & & $36(100)$ & & & & & & & & & & & & \\
\hline \multirow[t]{2}{*}{$\begin{array}{l}\text { Age } \\
(\text { mean } \\
\pm \mathrm{SD})\end{array}$} & & $58.8 \pm 8.6$ & $\begin{array}{c}9.5 \\
(2.5)\end{array}$ & $\begin{array}{c}1.1 \\
(4.6)\end{array}$ & $\begin{array}{l}10.9 \\
(2.8)\end{array}$ & $\begin{array}{c}6.4 \\
(2.6)\end{array}$ & $\begin{array}{l}1.08 \\
(2.8)\end{array}$ & $\begin{array}{c}7.8 \\
(4.4)\end{array}$ & $\begin{array}{c}2.2 \\
(1.5)\end{array}$ & $\begin{array}{l}1.99 \\
(1.7)\end{array}$ & $\begin{array}{c}1.1 \\
(8.5)\end{array}$ & $\begin{array}{l}3.77 \\
(4.4)\end{array}$ & $\begin{array}{c}3.4 \\
(3.1)\end{array}$ & $\begin{array}{c}5.8 \\
(3.8)\end{array}$ \\
\hline & ${ }^{\#} \mathbf{P}$ & & 0.54 & 0.12 & 0.48 & 0.27 & 0.007 & 0.24 & 0.43 & 0.16 & 0.46 & 0.60 & 0.84 & 0.84 \\
\hline \multirow[t]{2}{*}{$\begin{array}{l}\text { Gender } \\
(\mathrm{M} / \mathrm{F})\end{array}$} & & $24 / 12$ & $\begin{array}{c}9.8 \\
(2.9) / 1.0 \\
(2.9)\end{array}$ & $\begin{array}{l}1.09 \\
(3.7) \\
/ 1.1 \\
(4.6)\end{array}$ & $\begin{array}{c}12.2 \\
(9.1) \\
/ 8.6 \\
(30.7)\end{array}$ & $\begin{array}{c}8.2 \\
(3.7) \\
/ 5.2 \\
(2.3)\end{array}$ & $\begin{array}{c}1.1 \\
(6.8) \\
/ 9.2 \\
(7.6)\end{array}$ & $\begin{array}{c}8.8 \\
(6.9) \\
/ 4.8 \\
(2.4)\end{array}$ & $\begin{array}{c}2.3 \\
(2.9) / 2.5 \\
(1.8)\end{array}$ & $\begin{array}{c}1.5 \\
(1.18) \\
/ 2.2 \\
(1.9)\end{array}$ & $\begin{array}{l}1.09 \\
(9.3) \\
13.8 \\
(4.4)\end{array}$ & $\begin{array}{c}3.8 \\
(4.4) \\
17.04 \\
(6.8)\end{array}$ & $\begin{array}{c}2.8 \\
(2.8) \\
/ 7.02 \\
(6.02)\end{array}$ & $\begin{array}{c}5 \\
(4.2) \\
/ 5.4 \\
(4.6)\end{array}$ \\
\hline & $\mathbf{P}$ & & 0.6 & 0.75 & 0.25 & 0.01 & 0.14 & 0.03 & 0.39 & 0.26 & 0.95 & 0.14 & 0.03 & 0.86 \\
\hline \multirow[t]{5}{*}{ BMI } & $18.5-24.9$ & $9(25)$ & $\begin{array}{c}9.6 \\
(3.1)\end{array}$ & $\begin{array}{c}8.8 \\
(2.7)\end{array}$ & $\begin{array}{l}12.2 \\
(7.3)\end{array}$ & $\begin{array}{c}7.6 \\
(2.5)\end{array}$ & $\begin{array}{c}8.2 \\
(2.17)\end{array}$ & $\begin{array}{l}7.99 \\
(3.3)\end{array}$ & $\begin{array}{c}3.4 \\
(4.5)\end{array}$ & $\begin{array}{c}1.7 \\
(1.3)\end{array}$ & $\begin{array}{c}0.9 \\
(9.05)\end{array}$ & $\begin{array}{c}6.2 \\
(6.4)\end{array}$ & $\begin{array}{c}3.1 \\
(3.8)\end{array}$ & $\begin{array}{c}3.7 \\
(1.7)\end{array}$ \\
\hline & $25-30$ & $10(27.8)$ & $\begin{array}{l}1.07 \\
(2.2)\end{array}$ & $\begin{array}{c}1.4 \\
(5.2)\end{array}$ & $\begin{array}{l}10.3 \\
(3.5)\end{array}$ & $\begin{array}{c}7.8 \\
(4.9)\end{array}$ & $\begin{array}{c}1.2 \\
(07.08\end{array}$ & $\begin{array}{c}7.3 \\
(4.8)\end{array}$ & $\begin{array}{l}1.96 \\
(1.7)\end{array}$ & $\begin{array}{c}2.1 \\
(1.9)\end{array}$ & $\begin{array}{l}1.02 \\
(6.6)\end{array}$ & $\begin{array}{c}3.7 \\
(2.5)\end{array}$ & $\begin{array}{c}5.2 \\
(4.6)\end{array}$ & $\begin{array}{l}5.07 \\
(4.7)\end{array}$ \\
\hline & $>30$ & $1(2.78)$ & 8.4 & 1.38 & 5.5 & 3.6 & 1.38 & 3.7 & 2.8 & 1.6 & 0.5 & 1.7 & 5.4 & 5.1 \\
\hline & Missing & $16(44.4)$ & $9.8(2.9)$ & $\begin{array}{l}1.04 \\
(2.0)\end{array}$ & $\begin{array}{c}1.1 \\
(10.3)\end{array}$ & $\begin{array}{c}6.9 \\
(2.3)\end{array}$ & $\begin{array}{c}1.04 \\
(8.99)\end{array}$ & $\begin{array}{c}7.8 \\
(8.1)\end{array}$ & $\begin{array}{l}2.05 \\
(1.2)\end{array}$ & $\begin{array}{c}1.6 \\
(1.38)\end{array}$ & $\begin{array}{c}1.15 \\
(9.77)\end{array}$ & $\begin{array}{c}4.1 \\
(5.6)\end{array}$ & $\begin{array}{l}4.08 \\
(5.1)\end{array}$ & $\begin{array}{c}5.9 \\
(5.1)\end{array}$ \\
\hline & $\mathbf{P}$ & & 0.44 & 0.06 & 0.44 & 0.39 & 0.17 & 0.37 & 0.59 & 0.79 & 0.39 & 0.26 & 0.43 & 0.74 \\
\hline
\end{tabular}

\# Correlation $\mathrm{P}$ value between age and Adipokines levels at each time point

\subsection{Statistical analysis}

Mann-Whitney U test was used for comparing clinical and biochemical variables between groups. To assess differences between in-group variables, Chi-Square test was used. Spearman's correlation coefficients were estimated to determine associations between Adipokine concentrations and anthropometric measurements and biochemical variables. All statistical assessments were considered significant as P-value $<0.05$. All analyses were performed using SPSS version 16 (version 9.2; SAS Institute, Cary, NC).

\section{Results}

The demographical and clinical features of MI patients and Adipokine levels in the groups are shown in tables 1 and 2.

\subsection{Concentration of serum Adipokines in the studied time points}

There were no significant differences in serum Adiponectin and Leptin concentrations between the three time points in $\mathrm{MI}$ patients $(\mathrm{P}=0.3$ and $\mathrm{P}=0.3$, respectively; Figure 1$)$. However, a significant difference in serum Adipsin concentration between day 5 (T5) and day 180 (T180) post-MI was observed $(\mathrm{P}=0.02$, Fig. 1$)$. In addition, there was a significant difference in serum Resistin levels between day 0 (T0) and day 180 (T180) as well as day 5 (T5) and day $180(\mathrm{~T} 180)(\mathrm{P}=$ 0.0001 and $\mathrm{P}=0.009$, respectively, Fig. 1). Interestingly, the Adiponectin/Resistin ratio increased from T0 to T180 and the difference between $\mathrm{T} 180$ and $\mathrm{T} 0$ was significant $(\mathrm{P}=0.002)$.

\subsection{Concentration of Adipokines in the studied time points based on gender}

The serum Resistin concentration at T0 was significantly higher than $\mathrm{T} 180$ among women and men $(\mathrm{P}=0.004$, and $\mathrm{P}=$ 0.02 , respectively). A trend of decrease in Resistin level in both genders was observed from T0 to T180 as well (Figs. 2A and 2B). Adipsin T180 levels were higher in men than women $(\mathrm{P}=0.03$, Figure $2 \mathrm{C})$, while $\mathrm{T} 5$ Leptin levels were higher in women than men $(\mathrm{P}=0.03$, Fig. $2 \mathrm{C})$. No other significant difference was observed between Adipokine levels based on gender.

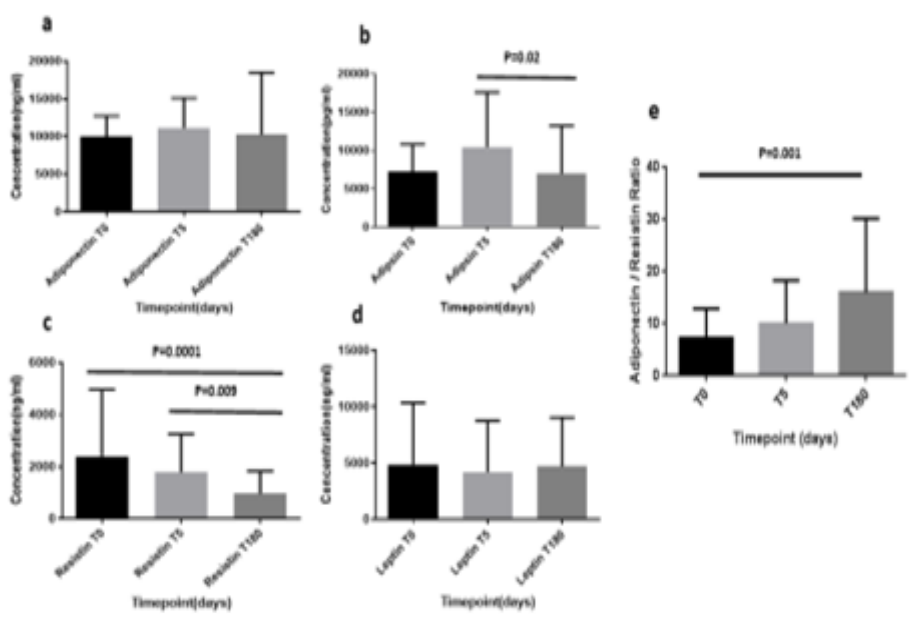

Fig. 1. Serum Adipokine levels in patients with MI at the time of admission to the hospital (T0), five days after admission (T5) and 180 days after MI (180). (a) Adiponectin levels (b) Adipsin levels, (c) Resistin levels, and (d) Leptin levels as well as Adiponectin to Resistin ratio (e) are shown 
Dowlatshahi et al. / J Exp Clin Med

Table 2. Adipokine levels based on clinical histories of AMI patients in each time point

\begin{tabular}{|c|c|c|c|c|c|c|c|c|c|c|c|c|c|c|}
\hline \multirow{2}{*}{\multicolumn{2}{|c|}{ Subjects }} & \multirow[t]{2}{*}{ No (\%) } & \multicolumn{3}{|c|}{$\begin{array}{l}\text { Adiponectin } \\
\text { Mean (SD) } \\
\left(\mathrm{ng} / \mathrm{ml} \times \mathbf{1 0}^{\mathbf{3}}\right)\end{array}$} & \multicolumn{3}{|c|}{$\begin{array}{c}\text { Adipsin } \\
\text { Mean (SD) } \\
\left(\mathbf{p g} / \mathbf{m l} \times 10^{\mathbf{3}}\right)\end{array}$} & \multicolumn{3}{|c|}{$\begin{array}{c}\text { Resistin } \\
\text { Mean (SD) } \\
\left(\mathbf{p g} / \mathbf{m l} \times \mathbf{1 0}^{\mathbf{3}}\right)\end{array}$} & \multicolumn{3}{|c|}{$\begin{array}{c}\text { Leptin } \\
\text { Mean (SD) } \\
\left(\mathbf{p g} / \mathbf{m l} \times 10^{\mathbf{3}}\right)\end{array}$} \\
\hline & & & T0 & T5 & T180 & T0 & T5 & T180 & T0 & T5 & T180 & T0 & T5 & T180 \\
\hline \multicolumn{15}{|l|}{$\mathrm{DM}$} \\
\hline & Pos & $9(25)$ & $\begin{array}{l}10.1 \\
(2.3)\end{array}$ & $\begin{array}{l}12.9 \\
(4.9)\end{array}$ & $\begin{array}{l}10.3 \\
(3.6)\end{array}$ & $\begin{array}{c}6.6 \\
(3.4)\end{array}$ & $\begin{array}{c}14.1 \\
(11.8)\end{array}$ & $\begin{array}{c}7.8 \\
(4.1)\end{array}$ & $\begin{array}{c}2 \\
(1.5)\end{array}$ & $\begin{array}{c}2.1 \\
(1.6)\end{array}$ & $\begin{array}{c}1.5 \\
(1.3)\end{array}$ & $\begin{array}{c}3.1 \\
(3.1)\end{array}$ & $\begin{array}{l}3.8 \\
(4.9)\end{array}$ & $\begin{array}{l}5.02 \\
(4.7)\end{array}$ \\
\hline & Neg & $27(75)$ & $\begin{array}{l}9.95 \\
(2.8)\end{array}$ & $\begin{array}{l}1.05 \\
(3.5)\end{array}$ & $\begin{array}{l}1.13 \\
(8.6)\end{array}$ & $\begin{array}{c}7.4 \\
(3.66)\end{array}$ & $\begin{array}{c}9.1 \\
(4.3)\end{array}$ & $\begin{array}{c}7.5 \\
(6.6)\end{array}$ & $\begin{array}{c}2.5 \\
(2.8)\end{array}$ & $\begin{array}{c}1.6 \\
(1.4)\end{array}$ & $\begin{array}{l}0.97 \\
(0.7)\end{array}$ & $\begin{array}{l}5.4 \\
(5.9)\end{array}$ & $\begin{array}{c}4.3 \\
(4.5)\end{array}$ & $\begin{array}{c}5.1 \\
(4.3)\end{array}$ \\
\hline & P value & & 0.91 & 0.12 & 0.69 & 0.66 & 0.77 & 0.60 & 0.94 & 0.31 & 0.34 & 0.19 & 0.59 & 0.98 \\
\hline \multicolumn{15}{|l|}{ HTN } \\
\hline & Pos & $20(55.6)$ & $\begin{array}{l}10.1 \\
(2.2)\end{array}$ & $\begin{array}{l}11.5 \\
(4.4)\end{array}$ & $\begin{array}{l}10.5 \\
(5.5)\end{array}$ & $\begin{array}{l}6.5 \\
(2.9)\end{array}$ & $\begin{array}{c}9.7 \\
(2.7)\end{array}$ & $\begin{array}{c}6.5 \\
(3.8)\end{array}$ & $\begin{array}{c}2.8 \\
(3.2)\end{array}$ & $\begin{array}{c}2.0 \\
(1.6)\end{array}$ & $\begin{array}{c}1.3 \\
(1.0)\end{array}$ & $\begin{array}{c}4.5 \\
(5.3)\end{array}$ & $\begin{array}{l}3.6 \\
(3.0)\end{array}$ & $\begin{array}{c}6.6 \\
(4.9)\end{array}$ \\
\hline & $\mathrm{Neg}$ & $16(44.4)$ & $\begin{array}{c}9.8 \\
(3.2)\end{array}$ & $\begin{array}{l}10.5 \\
(3.5)\end{array}$ & $\begin{array}{c}12 \\
(10.3)\end{array}$ & $\begin{array}{c}8.1 \\
(4.1)\end{array}$ & $\begin{array}{l}11.3 \\
(7.1)\end{array}$ & $\begin{array}{l}8.8 \\
(8.03)\end{array}$ & $\begin{array}{l}1.8 \\
(1.1)\end{array}$ & $\begin{array}{c}1.5 \\
(1.1)\end{array}$ & $\begin{array}{c}0.7 \\
(0.4)\end{array}$ & $\begin{array}{l}5.2 \\
(5.9)\end{array}$ & $\begin{array}{c}4.9 \\
(5.9)\end{array}$ & $\begin{array}{c}3.3 \\
(2.6)\end{array}$ \\
\hline & P value & & 0.88 & 0.60 & 0.92 & 0.24 & 0.42 & 0.53 & 0.47 & 0.51 & 0.25 & 0.88 & 0.88 & 0.04 \\
\hline \multicolumn{15}{|l|}{ HLP } \\
\hline & Pos & $9(25)$ & $\begin{array}{c}9.2 \\
(2.2)\end{array}$ & $\begin{array}{l}12.4 \\
(3.3)\end{array}$ & $\begin{array}{l}12.8 \\
(13.3)\end{array}$ & $\begin{array}{l}8.07 \\
(3.2)\end{array}$ & $\begin{array}{l}15.0 \\
(9.6)\end{array}$ & $\begin{array}{c}9.3 \\
(10.2)\end{array}$ & $\begin{array}{c}1.7 \\
(1.4)\end{array}$ & $\begin{array}{c}1.9 \\
(1.6)\end{array}$ & $\begin{array}{c}0.8 \\
(0.4)\end{array}$ & $\begin{array}{l}4.7 \\
(5.1)\end{array}$ & $\begin{array}{c}4.6 \\
(3.8)\end{array}$ & $\begin{array}{c}6.4 \\
(4.3)\end{array}$ \\
\hline & Neg & $27(75)$ & $\begin{array}{l}10.2 \\
(2.8)\end{array}$ & $\begin{array}{l}10.6 \\
(4.1)\end{array}$ & $\begin{array}{l}10.5 \\
(4.9)\end{array}$ & $\begin{array}{c}6.9 \\
(3.7)\end{array}$ & $\begin{array}{c}8.9 \\
(5.4)\end{array}$ & $\begin{array}{c}6.9 \\
(3.7)\end{array}$ & $\begin{array}{c}2.6 \\
(2.8)\end{array}$ & $\begin{array}{c}1.7 \\
(1.4)\end{array}$ & $\begin{array}{c}1.1 \\
(0.9)\end{array}$ & $\begin{array}{c}4.9 \\
(5.7)\end{array}$ & $\begin{array}{c}4.1 \\
(4.8)\end{array}$ & $\begin{array}{c}4.6 \\
(4.2)\end{array}$ \\
\hline & $P$ value & & 0.33 & 0.10 & 0.61 & 0.36 & 0.06 & 0.95 & 0.21 & 0.64 & 0.85 & 0.80 & 0.29 & 0.19 \\
\hline \multicolumn{15}{|l|}{$\begin{array}{l}\text { Smok } \\
\text { ing }\end{array}$} \\
\hline & Yes & $22(61.1)$ & $\begin{array}{l}10.6 \\
(2.7)\end{array}$ & $\begin{array}{l}11.9 \\
(4.3)\end{array}$ & $\begin{array}{c}12.7 \\
(9.2)\end{array}$ & $\begin{array}{c}7.6 \\
(3.1)\end{array}$ & $\begin{array}{l}11.1 \\
(8.2)\end{array}$ & $\begin{array}{c}8.7 \\
(6.9)\end{array}$ & $\begin{array}{c}2.4 \\
(3.1)\end{array}$ & $\begin{array}{l}1.5 \\
(1.2)\end{array}$ & $\begin{array}{c}1.2 \\
(0.9)\end{array}$ & $\begin{array}{c}3.1 \\
(4.1)\end{array}$ & $\begin{array}{l}2.7 \\
(3.0)\end{array}$ & $\begin{array}{c}5.7 \\
(4.9)\end{array}$ \\
\hline & No & $14(38.9)$ & $\begin{array}{c}9.0 \\
(2.4)\end{array}$ & $\begin{array}{c}9.8 \\
(3.0)\end{array}$ & $\begin{array}{c}8.1 \\
(2.9)\end{array}$ & $\begin{array}{c}6.6 \\
(4.2)\end{array}$ & $\begin{array}{c}9.4 \\
(5.2)\end{array}$ & $\begin{array}{c}5.4 \\
(3.4)\end{array}$ & $\begin{array}{c}2.3 \\
(1.4)\end{array}$ & $\begin{array}{c}2.2 \\
(1.8)\end{array}$ & $\begin{array}{c}0.7 \\
(0.3)\end{array}$ & $\begin{array}{c}7.5 \\
(6.3)\end{array}$ & $\begin{array}{c}6.5 \\
(5.6)\end{array}$ & $\begin{array}{c}4.0 \\
(2.4)\end{array}$ \\
\hline & P value & & 0.09 & 0.17 & 0.04 & 0.15 & 0.73 & 0.06 & 0.25 & 0.19 & 0.37 & 0.01 & 0.01 & 0.66 \\
\hline \multicolumn{15}{|l|}{ VD } \\
\hline & CAD & $6(16.7)$ & $\begin{array}{l}12.1 \\
(2.3)\end{array}$ & $\begin{array}{l}11.7 \\
(3.8)\end{array}$ & $\begin{array}{l}10.1 \\
(2.9)\end{array}$ & $\begin{array}{c}7.9 \\
(4.2)\end{array}$ & $\begin{array}{c}12.6 \\
(11.3)\end{array}$ & $\begin{array}{c}5.5 \\
(3.1)\end{array}$ & $\begin{array}{c}1.8 \\
(1.4)\end{array}$ & $\begin{array}{c}1.1 \\
(0.3)\end{array}$ & $\begin{array}{c}0.7 \\
(2.3)\end{array}$ & $\begin{array}{c}4.5 \\
(7.5)\end{array}$ & $\begin{array}{c}4.4 \\
(4.3)\end{array}$ & $\begin{array}{c}6.2 \\
(3.8)\end{array}$ \\
\hline & No VD & $30(83.3)$ & $\begin{array}{c}9.6 \\
(2.6)\end{array}$ & $\begin{array}{l}10.9 \\
(4.1)\end{array}$ & $\begin{array}{l}11.4 \\
(8.7)\end{array}$ & $\begin{array}{c}7.1 \\
(3.5)\end{array}$ & $\begin{array}{l}10.0 \\
(6.2)\end{array}$ & $\begin{array}{c}8.1 \\
(6.5)\end{array}$ & $\begin{array}{c}2.5 \\
(2.7)\end{array}$ & $\begin{array}{c}1.9 \\
(1.5)\end{array}$ & $\begin{array}{c}1.1 \\
(9.1)\end{array}$ & $\begin{array}{c}4.9 \\
(5.1)\end{array}$ & $\begin{array}{c}4.1 \\
(4.6)\end{array}$ & $\begin{array}{c}4.9 \\
(4.4)\end{array}$ \\
\hline & P value & & 0.07 & 0.53 & 0.76 & 0.51 & 0.89 & 0.30 & 0.28 & 0.29 & 0.51 & 0.36 & 0.83 & 0.20 \\
\hline \multicolumn{15}{|l|}{ HR } \\
\hline & $\begin{array}{c}\text { Normal } \\
(60-100 \\
\text { bpm })\end{array}$ & $24(66.7)$ & $\begin{array}{c}9.7 \\
(2.9)\end{array}$ & $\begin{array}{l}10.6 \\
(3.8)\end{array}$ & $\begin{array}{l}11.6 \\
(9.1)\end{array}$ & $\begin{array}{l}8.01 \\
(3.7)\end{array}$ & $\begin{array}{l}11.0 \\
(7.4)\end{array}$ & $\begin{array}{c}8.3 \\
(6.8)\end{array}$ & $\begin{array}{c}2.6 \\
(2.9)\end{array}$ & $\begin{array}{c}1.7 \\
(1.4)\end{array}$ & $\begin{array}{c}0.9 \\
(0.7)\end{array}$ & $\begin{array}{c}5.1 \\
(5.8)\end{array}$ & $\begin{array}{l}4.5 \\
(5.1)\end{array}$ & $\begin{array}{l}4.5 \\
(4.0)\end{array}$ \\
\hline & $\begin{array}{c}\text { Abnormal } \\
\text { (ELSE) }\end{array}$ & $12(33.3)$ & $\begin{array}{l}10.5 \\
(2.1)\end{array}$ & $\begin{array}{l}12.1 \\
(4.3)\end{array}$ & $\begin{array}{c}9.9 \\
(3.1)\end{array}$ & $\begin{array}{c}5.6 \\
(2.7)\end{array}$ & $\begin{array}{c}9.2 \\
(6.6)\end{array}$ & $\begin{array}{l}5.5 \\
(2.9)\end{array}$ & $\begin{array}{c}1.9 \\
(1.4)\end{array}$ & $\begin{array}{c}1.9 \\
(1.5)\end{array}$ & $\begin{array}{c}1.2 \\
(1.1)\end{array}$ & $\begin{array}{c}4.3 \\
(4.7)\end{array}$ & $\begin{array}{c}3.5 \\
(3.3)\end{array}$ & $\begin{array}{c}6.6 \\
(4.8)\end{array}$ \\
\hline & $P$ value & & 0.43 & 0.19 & 0.86 & 0.06 & 0.46 & 0.27 & 0.73 & 0.48 & 0.65 & 0.81 & 0.91 & 0.25 \\
\hline \multicolumn{15}{|l|}{ SBP } \\
\hline & $\begin{array}{c}\text { Normal } \\
(<150 \\
\mathrm{mmHg})\end{array}$ & $19(53)$ & $\begin{array}{c}9.9 \\
(2.9)\end{array}$ & $\begin{array}{c}10.8 \\
(303)\end{array}$ & $\begin{array}{l}11.6 \\
(10)\end{array}$ & $\begin{array}{l}6.4 \\
(3.1)\end{array}$ & $\begin{array}{c}9.9 \\
(7.2)\end{array}$ & $\begin{array}{c}8.8 \\
(7.8)\end{array}$ & $\begin{array}{c}2.7 \\
(3.2)\end{array}$ & $\begin{array}{c}2 \\
(1.6)\end{array}$ & $\begin{array}{l}8.5 \\
(8.3)\end{array}$ & $\begin{array}{c}5.8 \\
(6.7)\end{array}$ & $\begin{array}{c}3.8 \\
(4.2)\end{array}$ & $\begin{array}{l}3.9 \\
(2.5)\end{array}$ \\
\hline & $\begin{array}{c}\text { Abnormal } \\
\text { (ELSE) }\end{array}$ & $17(47)$ & $\begin{array}{l}10.1 \\
(2.5)\end{array}$ & $\begin{array}{l}11.1 \\
(4.7)\end{array}$ & $\begin{array}{l}10.8 \\
(5.6)\end{array}$ & $\begin{array}{c}8.2 \\
(3.9)\end{array}$ & $\begin{array}{l}11.0 \\
(7.3)\end{array}$ & $\begin{array}{c}6.5 \\
(3.9)\end{array}$ & $\begin{array}{c}2.0 \\
(1.5)\end{array}$ & $\begin{array}{c}1.5 \\
(1.3)\end{array}$ & $\begin{array}{l}1.3 \\
(0.8)\end{array}$ & $\begin{array}{c}3.9 \\
(3.6)\end{array}$ & $\begin{array}{c}4.6 \\
(5.0)\end{array}$ & $\begin{array}{c}6.3 \\
(5.3)\end{array}$ \\
\hline & $P$ value & & 0.66 & 1.0 & 0.71 & 0.21 & 0.51 & 0.49 & 0.66 & 0.14 & 0.02 & 0.75 & 0.49 & 0.22 \\
\hline \multicolumn{15}{|l|}{$\mathrm{RR}$} \\
\hline & $\begin{array}{c}\text { Normal } \\
(12-20 \\
\text { bpm) }\end{array}$ & $16(44.4)$ & $\begin{array}{c}9.9 \\
(2.3)\end{array}$ & $\begin{array}{l}11.1 \\
(4.2)\end{array}$ & $\begin{array}{l}10.8 \\
(6.4)\end{array}$ & $\begin{array}{c}5.6 \\
(2.5)\end{array}$ & $\begin{array}{c}8.8 \\
(6.6)\end{array}$ & $\begin{array}{l}6.03 \\
(4.2)\end{array}$ & $\begin{array}{c}2.5 \\
(1.6)\end{array}$ & $\begin{array}{c}1.7 \\
(1.4)\end{array}$ & $\begin{array}{c}1.1 \\
(8.6)\end{array}$ & $\begin{array}{l}6.07 \\
(6.3)\end{array}$ & $\begin{array}{c}6.1 \\
(5.9)\end{array}$ & $\begin{array}{c}4.5 \\
(4.2)\end{array}$ \\
\hline & $\begin{array}{c}\text { Abnormal } \\
\text { (ELSE) }\end{array}$ & $20(55.6)$ & $\begin{array}{c}10 \\
(3.02)\end{array}$ & $\begin{array}{l}11.1 \\
(3.9)\end{array}$ & $\begin{array}{l}11.3 \\
(9.01)\end{array}$ & $\begin{array}{c}8.5 \\
(3.8)\end{array}$ & $\begin{array}{l}11.1 \\
(7.4)\end{array}$ & $\begin{array}{l}8.6 \\
(7.02)\end{array}$ & $\begin{array}{c}2.3 \\
(3.1)\end{array}$ & $\begin{array}{c}1.8 \\
(1.5)\end{array}$ & $\begin{array}{l}1.01 \\
(8.5)\end{array}$ & $\begin{array}{c}3.9 \\
(4.6)\end{array}$ & $\begin{array}{c}2.6 \\
(2.1)\end{array}$ & $5.5(4.3)$ \\
\hline & $P$ value & & 0.79 & 0.96 & 0.89 & 0.03 & 0.09 & 0.15 & 0.19 & 0.84 & 0.65 & 0.42 & 0.24 & 0.43 \\
\hline Total & & $36(100)$ & & & & & & & & & & & & \\
\hline
\end{tabular}




\subsection{Concentration of Adipokines in the studied time points based on smoking status}

Serum Adiponectin concentration at all three time-points was higher in smokers compared with non-smokers, but only at T180 the difference reached the significant level $(\mathrm{P}=0.04$, Fig. 3A). The levels of Adipsin were also non-significantly higher in smokers than non-smokers (Fig. 3B). Serum Resistin levels varied over time and was only non-significantly higher in non-smokers at T5. Leptin levels were higher in non-smokers than smokers, but the difference reached a significantly higher level only at $\mathrm{T} 0$ and $\mathrm{T} 5 \quad(\mathrm{P}=0.01$ and $\mathrm{P}=0.01$, respectively, Figs. 3C and D).

\subsection{Correlation of Adipokine levels with risk factors and clinical and demographical characteristics of patients} In bivariate correlation analyses, serum Adipsin concentration at T0 was found to be significantly and inversely correlated with heart rate, respiratory rate and pulse rate $(\mathrm{P}=0.01, \mathrm{r}=-$ 0.39 ,

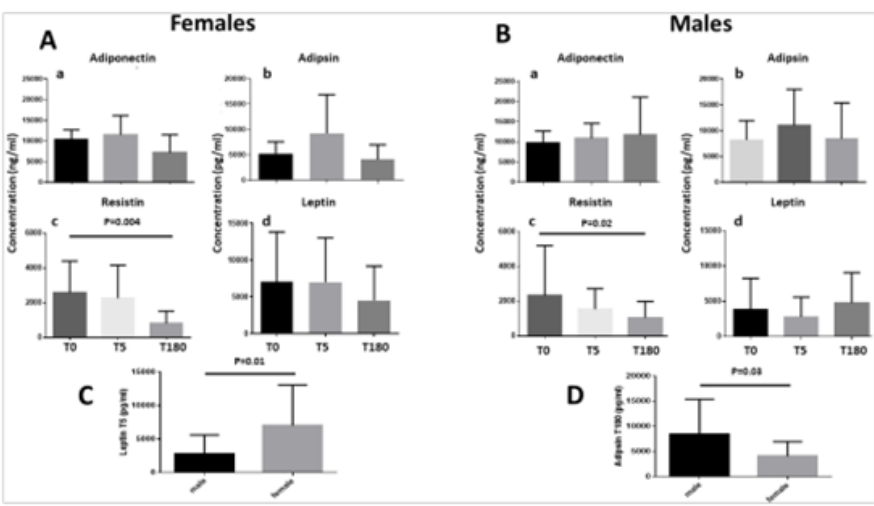

Fig. 2. Concentration of adipokines in the studies time points based on gender. Adiponectin (a), Adipsin (b), Resistin (c) and Leptin (d) in Females (A) and (B) Males. Differences in the Leptin T5 (C) and Adipsin T180 (D) between genders were statistically significant $(\mathrm{P}<0.05)$

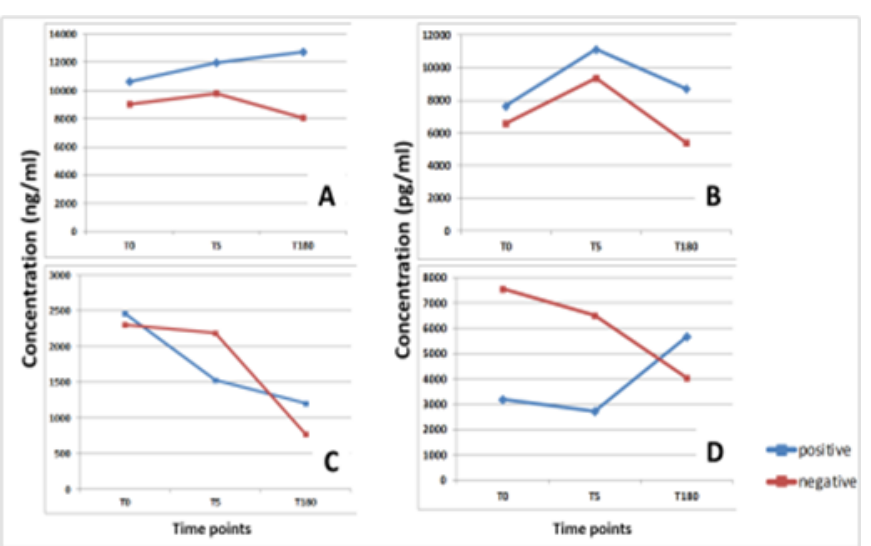

Fig. 3. Concentration of adipokines in the studies time points based on smoking status. Trends of changes in (A) Adiponectin, (B) Adipsin, (C) Resistin, and (D) Leptin in sera of patients

$\mathrm{P}=0.01, \mathrm{r}=-0.40$ and $\mathrm{r}=-0.35, \mathrm{p}=0.03$, respectively; Figs. 4A, 4B and 4C). Adipsin T5 levels were significantly and inversely correlated with age $(\mathrm{r}=-0.44, \mathrm{P}=0.007$, Fig. 4D). In addition, serum Leptin concentration at T5 and serum
Resistin concentration at T180 were lower in those patients who had atrioventricular (AV) block $(\mathrm{P}=0.04$ and $\mathrm{P}=0.05$ respectively; Fig.5 A, B). Anteroseptal Hypokinesia correlated with serum Resistin level at T0 $(\mathrm{P}=0.02)$ and serum Adipsin level at T5 $(\mathrm{P}=0.02)$ while Leptin at T0 and T5 correlated with Anterolateral Hypokinesia $(\mathrm{P}=0.03$ and $\mathrm{P}=0.004$, respectively; Figs. 5 C-F).
A

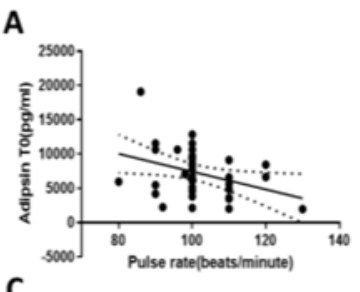

C

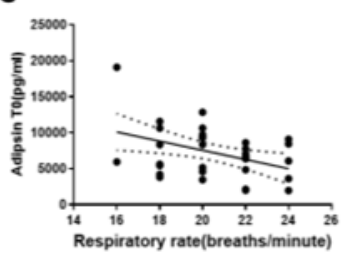

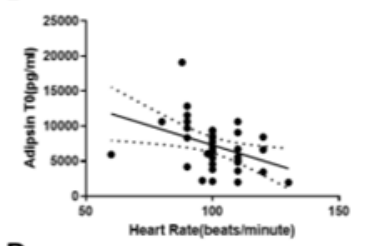

D

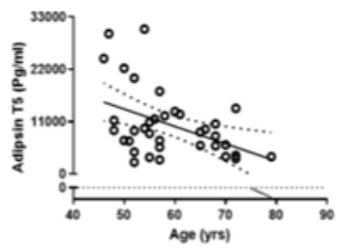

Fig. 4. Correlation of serum Adipsin concentration at $\mathrm{T} 0$ with heart rate, respiratory rate and pulse rate as well as Adipsin T5 with age in patients
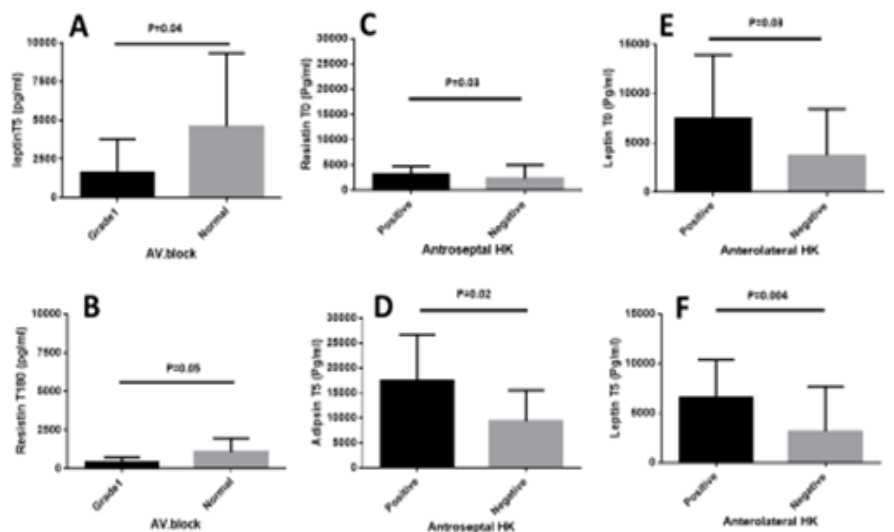

Fig. 5. Comparison of adipokine levels between patients. (A, B) Leptin levels at T5 and Resistin levels at T180 in patients with Atrioventricular (AV) block as compared to patients without block, C) Resistin T0 levels, D) Adipsin T5 levels, E) Leptin T0 levels and F) Leptin T5 levels in patients with Anteroseptal hypokinesia and those without

A significant negative correlation was observed between Adiponectin/Resistin ratio at $\mathrm{T} 180$ and Respiratory rate ( $\mathrm{r}=$ 0.35, $\mathrm{P}=0.048$, Fig. 6A). Moreover, Adiponectin/Resistin ratio at T180 was significantly higher in patients with $\mathrm{AV}$ block grade- 1 compared to those without AV block $(\mathrm{P}=0.025$, Fig. 6B). Adiponectin/Resistin ratio at $\mathrm{T} 180$ was, however, lower in patients with Anterolateral Hypokinesia $(\mathrm{P}=0.024$, Fig. 6C). Moreover, systolic blood pressure was found to be negatively correlated with serum Resistin $(\mathrm{P}=0.04, \mathrm{r}=-0.34)$ and positively with serum Leptin concentrations at T180 $(\mathrm{P}=0.01, \mathrm{r}=0.40$, Fig. 7$)$. 
A

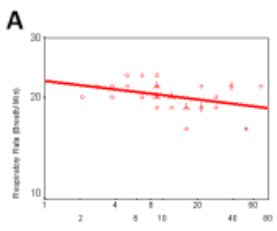

B

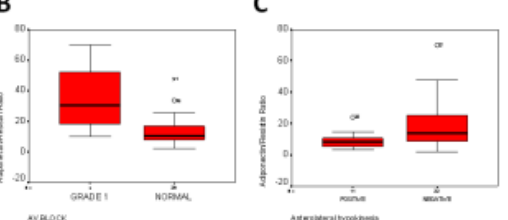

Fig. 6. Correlation between Adiponectin/Resistin ratio (at T180) and Respiratory rate (A), Atrioventricular (AV) block (B), and Anterolateral Hypokinesia (C) in patients with MI

\subsection{Comparison of Adipokine levels between patients based on their Acetylsalicylic Acid (ASA) and Statin treatments}

We also compared the Adipokine levels between patients who had received ASA and/or Statin therapy in each time point. As shown in Table 3, there was no significant difference in the level of Adipokines between patients who received ASA
A

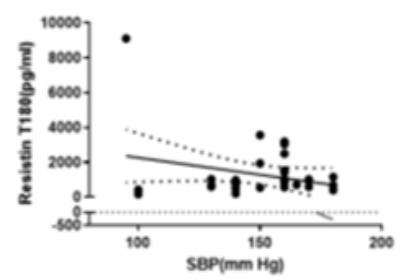

B

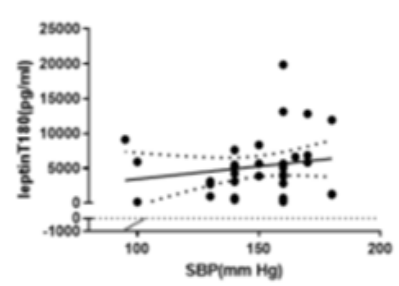

Fig. 7. Correlation of serum Resistin and Leptin concentrations at T180 with systolic blood pressure in patients

and those who did not in any time point. For Statin, the only significant difference was observed in Resistin levels where those patients who had received Statin therapy had lower Resistin levels at the time of admission to the hospital (T0) compared to those who were not on Statin therapy $(\mathrm{P}=0.014)$ (Table 4).

Table 3. Adipokine levels in each time point based on ASA therapy

\begin{tabular}{|c|c|c|c|c|c|}
\hline \multicolumn{6}{|c|}{ ASA } \\
\hline \multicolumn{3}{|c|}{ Time point } & T0 & T5 & T180 \\
\hline \multirow{3}{*}{ Adiponectin } & \multicolumn{2}{|c|}{ P. value } & 0.074 & 0.077 & 0.432 \\
\hline & \multirow{2}{*}{$\begin{array}{l}\text { Mean } \\
\left.\times 10^{3}\right)\end{array}$} & Pos. $(N=14)$ & $11.1 \pm 2.3$ & $12.08 \pm 3.6$ & $13.1 \pm 11.4$ \\
\hline & & Neg. $(N=22)$ & $9.2 \pm 2.7$ & $10 \pm 4.1$ & $10 \pm 5.1$ \\
\hline \multirow{3}{*}{ Adipsin } & \multicolumn{2}{|c|}{ P. value } & 0.745 & 0.465 & 0.210 \\
\hline & \multirow{2}{*}{$\begin{array}{l}\text { Mean } \\
\left(\mathrm{pg} / \mathrm{ml} \times 10^{3}\right)\end{array}$} & Pos. $(N=14)$ & $7.3 \pm 3.5$ & $9.8 \pm 7.9$ & $10.2 \pm 8.8$ \\
\hline & & Neg. $(N=22)$ & $7.1 \pm 3.6$ & $10.8 \pm 6.7$ & $6.1 \pm 3.1$ \\
\hline \multirow{3}{*}{ Leptin } & \multicolumn{2}{|c|}{ P. value } & 0.236 & 0.626 & 0.389 \\
\hline & \multirow{2}{*}{$\begin{array}{l}\text { Mean } \\
\left(\mathrm{pg} / \mathrm{ml} \times 10^{3}\right)\end{array}$} & Pos. $(N=14)$ & $4.3 \pm 6.1$ & $3.06 \pm 2.4$ & $5.4 \pm 3.1$ \\
\hline & & Neg. $(N=22)$ & $5.2 \pm 5.1$ & $4.9 \pm 5.4$ & $4.9 \pm 4.9$ \\
\hline \multirow{3}{*}{ Resistin } & \multicolumn{2}{|c|}{ P. value } & 0.390 & 0.795 & 0.837 \\
\hline & \multirow{2}{*}{$\begin{array}{l}\text { Mean } \\
\left(\mathrm{pg} / \mathrm{ml} \times 10^{3}\right)\end{array}$} & Pos. $(\mathrm{N}=14)$ & $2.5 \pm 3.6$ & $1.9 \pm 1.5$ & $1.2 \pm 1.1$ \\
\hline & & Neg. $(N=22)$ & $2.3 \pm 1.6$ & $1.7 \pm 1.4$ & $0.9 \pm 0.6$ \\
\hline
\end{tabular}

Table 4. Adipokine levels in each time point based on Statin therapy

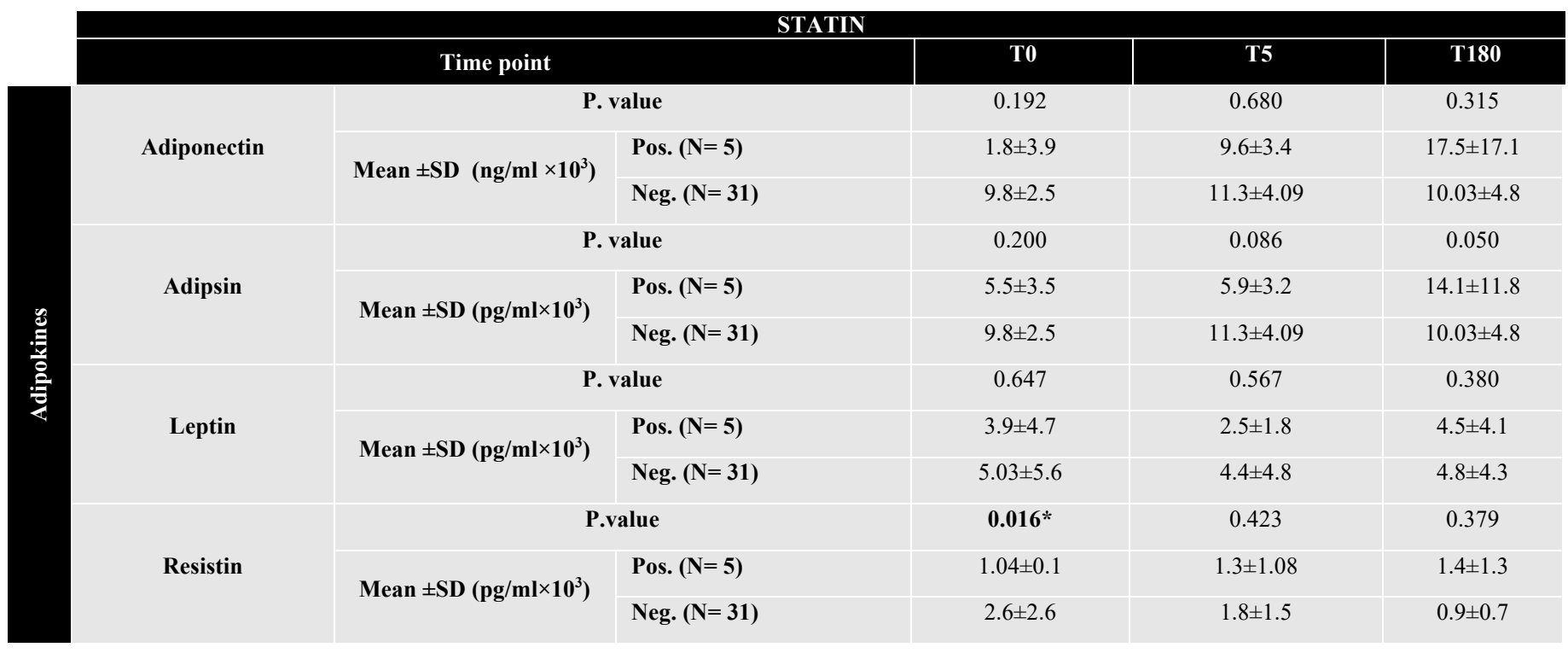




\section{Discussion}

It is shown that Adult cardiomyocytes produce Adipokines to protect the damaged cardiomyocytes (27). In our study, the serum concentrations of Adiponectin, Adipsin, Resistin and Leptin were measured in patients with MI at three different time points: during the first 12 hours of hospitalization (T0), five days after hospitalization (T5), and after six months of MI (T180). Our results indicated that Adipokines concentrations show various trends over time. Comparison of serum Adiponectin and Leptin concentrations at the three time points showed no significant changes over time. Resistin decreased significantly over the six-month follow-up and Adipsin levels fluctuated between time points. A previous study has shown that Resistin accelerates mechanisms involved in production of inflammatory cytokines $(28,29)$. Resistin increases p38 MAPK phosphorylation and NF-kappa B expression, however, anti-inflammatory treatments after MI, such as Aspirin, may decrease the effect of Resistin $(28,29)$. In our hands, however, there was no difference in the Resistin levels between those who were treated with Aspirin and those who were not in any of the time points. For Statin, however, we found that those patients who had received Statin therapy before MI had lower Resistin levels at the time of admission to the hospital (T0) compared to those who did not. This difference, however, should be interpreted with caution as the number of cases in the Statin treated group was very low $(n=5)$. In accordance with studies in polycystic ovary syndrome (30) and acute coronary syndrome (31) we observed an increasing trend in Adiponectin to Resistin ratio after six months.

Resistin decrease started earlier in men compared to women after incidence of MI (Fig. 2). Previously, it was shown that Resistin is higher in women with diabetes than men with diabetes (32). A previous study on a small group of obese healthy individuals, has shown a higher level of Adipokines, including Resistin in females than males (33). Of note, Resistin is not associated with menstruation and does not change significantly during this period $(34,35)$.

In our study, there was a significant inverse relationship between smoking and serum Leptin concentration at earlier time points. This finding is not new and is in accordance with several previous studies $(36,37)$. However, there are contradictory reports that state otherwise in individuals with MI and atherosclerosis $(38,39)$. Knowing that after the first myocardial infarction, smokers have a shorter life expectancy because they may suffer from stroke at a younger age (40), and that smoking independently predicts major cardiovascular events, heart failure, and mortality (41), our results on the correlation of serum Leptin levels five days after MI as well as serum Adiponectin levels six months after MI with smoking may be important.

Interestingly, higher Adipsin levels at T0 were associated with decreased heart rate, decreased pulse rate, and decreased respiratory rate. A correlation between Adipsin and heart failure is reported (42), and Adipsin is suggested as one of the single predictors of heart failure (43). A recent study on a cohort of 370 patients with atherosclerosis showed that patients with higher Adipsin levels had a 4.2 fold increase in all-cause death and 2.4 fold increase in rehospitalisation (44). Interestingly, four of the five patients who developed MI during their course of follow-up and died because of MI had Adipsin levels higher than $400 \mathrm{ng} / \mathrm{ml}$ (44).

We also found that Leptin T5 and Resistin T180 were associated with atrioventricular block which is in accordance with previous studies showing the significance of these Adipokines in Atrial Fibrillation associated disorders (45-47).

We also found that Leptin levels were higher in patients with Anterolateral Hypokinesia in T0 and T5. Previous studies have shown that BMI correlates with left ventricular diastolic dysfunction (48), however, it decreases global hypokinesia of the heart (49). Bearing in mind that in addition to being an obesity related Adipokine, Leptin is also an inflammatory cytokine which contributes to other pathways and various diseases, the mechanistic involvement and significance of Leptin in Anterolateral Hypokinesia remains to be investigated $(8,10)$. Our results also showed that Anteroseptal Hypokinesia was associated with Resistin T0 and Adipsin T5 levels. These finding may be related to the cross-talk between heart adipose tissue and heart myocytes through these Adipokines similar to what is seen for Adiponectin (50). The lower ratios of Adiponectin/Resistin at T180 in patients with Anterolateral Hypokinesia may indicate a beneficial effect of Adiponectin on myocytes as previously suggested (50). Interestingly, the Adiponectin/Resistin ratio at T180 negatively correlated with respiratory rate at the time of admission, indicating the negative correlation of this index with MI prognosis.

Our results indicate that like other cytokines, Adipokines play their role in a network and may have both beneficial and harmful effect which is balanced by other Adipokines. Finding their interaction and their mechanism of action may pave the way for finding new cardiac biomarkers and/or drug targets.

\section{Conflict of interest}

The author(s) declare no conflicts of interest.

\section{Acknowledgments}

This work was performed as part of Ehsan Dowlatshahi (M.Sc. of Immunology) dissertation as a requirement for graduation from Shiraz School of Medicine (Shiraz, Iran). This project was financially supported by a grant (97-16921) from Shiraz University of Medical Sciences, Shiraz, Iran. The code of ethical approval of this project is IR.SUMS.REC.1397.1096. No writing assistance was utilized in the production of this manuscript. 


\section{References}

1. Benjamin EJ, Muntner P, Alonso A, Bittencourt MS, Callaway CW, Carson AP, et al. Heart Disease and Stroke Statistics-2019 Update: A Report From the American Heart Association. Circulation. 2019;139(10):e56-e528.

2. Abbate A, Bussani R, Sinagra G, Barresi E, Pivetta A, Perkan A, et al. Right Ventricular Cardiomyocyte Apoptosis in Patients With Acute Myocardial Infarction of the Left Ventricular Wall. Am J Cardiol. 2008;102(6):658-62.

3. Granger DN, Rodrigues SF, Yildirim A, Senchenkova EY. Microvascular responses to cardiovascular risk factors. Microcirculation. 2010;17(3):192-205.

4. Fasshauer M, Bluher M. Adipokines in health and disease. Trends Pharmacol Sci. 2015;36(7):461-70.

5. Gualillo O. Elevated serum leptin concentrations induced by experimental acute inflammation. Life Sciences 2000;67:243341.

6. Procaccini C, La Rocca C, Carbone F, De Rosa V, Galgani M, Matarese G. Leptin as immune mediator: Interaction between neuroendocrine and immune system. Dev Comp Immunol. 2017;66:120-9.

7. Kontunen $\mathrm{P}$, Vuolteenaho $\mathrm{K}$, Nieminen $\mathrm{R}$, Lehtimaki L, Kautiainen H, Kesaniemi $\mathrm{Y}$, et al. Resistin is linked to inflammation, and leptin to metabolic syndrome, in women with inflammatory arthritis. Scand J Rheumatol. 2011;40(4):256-62.

8. Mancuso P. The role of adipokines in chronic inflammation. Immunotargets Ther. 2016;5:47-56.

9. Lehr S, Hartwig S, Sell H. Adipokines: a treasure trove for the discovery of biomarkers for metabolic disorders. Proteomics Clin Appl. 2012;6(1-2):91-101.

10. Bluher M. Adipokines removing road blocks to obesity and diabetes therapy. Mol Metab. 2014;3(3):230-40.

11. Smekal A, Vaclavik J. Adipokines and cardiovascular disease: A comprehensive review. Biomed Pap Med Fac Univ Palacky Olomouc Czech Repub. 2017;161(1):31-40.

12. Ekmen N, Helvaci A, Gunaldi M, Sasani H, Yildirmak ST. Leptin as an important link between obesity and cardiovascular risk factors in men with acute myocardial infarction. Indian Heart J. 2016;68(2):132-7.

13. Muse ED. The Association of Resistin with Cardiovascular Disease in The Multi-Ethnic Study of Atherosclerosis. Atherosclerosis. 2015;239(1):101-8.

14. Lehrke M, Reilly MP, Millington SC, Iqbal N, Rader DJ, Lazar MA. An inflammatory cascade leading to hyperresistinemia in humans. PLoS Med. 2004;1(2):e45.

15. Aquilante CL, Kosmiski LA, Knutsen SD, Zineh I. Relationship between plasma resistin concentrations, inflammatory chemokines, and components of the metabolic syndrome in adults. Metabolism. 2008;57(4):494-501.

16. Ntaios G, Gatselis NK, Makaritsis K, Dalekos GN. Adipokines as mediators of endothelial function and atherosclerosis. Atherosclerosis. 2013;227(2):216-21.

17. Kim M, Oh JK, Sakata S, Liang I, Park W, Hajjar RJ, et al. Role of resistin in cardiac contractility and hypertrophy. J Mol Cell Cardiol. 2008;45(2):270-80.

18. Kaser S, Kaser A, Sandhofer A, Ebenbichler CF, Tilg H, Patsch JR. Resistin messenger-RNA expression is increased by proinflammatory cytokines in vitro. Biochem Biophys Res Commun. 2003;309(2):286-90.
19. Calabro P, Samudio I, Willerson JT, Yeh ET. Resistin promotes smooth muscle cell proliferation through activation of extracellular signal-regulated kinase $1 / 2$ and phosphatidylinositol 3-kinase pathways. Circulation. 2004;110(21):3335-40.

20. Kawanami D, Maemura $K$, Takeda $N$, Harada T, Nojiri T, Imai $\mathrm{Y}$, et al. Direct reciprocal effects of resistin and adiponectin on vascular endothelial cells: a new insight into adipocytokineendothelial cell interactions. Biochem Biophys Res Commun. 2004;314(2):415-9.

21. Scherer PE. A Novel Serum Protein Similar to C1q, Produced Exclusively in adipocyte. J Biol Chem. 1995;45(10).

22. Hu E. AdipoQ Is a Novel Adipose-specific Gene Dysregulated in Obesity. J Biol Chem. 1996;271(18):10697-703.

23. Pyrzak B, M. Ruminska, K. Popko, Demkow U. Adiponectin as a biomarker of the metabolic syndrome in children and adolescents. Eur J Med Res. 2010;15.

24. Murohara RSNOT. Adiponectin and Cardiovascular Disease. Circ J. 2009;73:608-14.

25. Luc G, Empana JP, Morange P, Juhan-Vague I, Arveiler D, Ferrieres $\mathrm{J}$, et al. Adipocytokines and the risk of coronary heart disease in healthy middle aged men: the PRIME Study. Int $\mathbf{J}$ Obes (Lond). 2010;34(1):118-26.

26. Cianflone K, Xia Z, Chen LY. Critical review of acylationstimulating protein physiology in humans and rodents. Biochimica et Biophysica Acta (BBA) - Biomembranes. 2003;1609(2):127-43.

27. Piñeiro R, Iglesias MJ, Gallego R, Raghay K, Eiras S, Rubio J, et al. Adiponectin is synthesized and secreted by human and murine cardiomyocytes. FEBS letters. 2005;579(23):5163-9.

28. Ou H-C, Lee W-J, Wu C-M, Chen JF-M, Sheu WH-H. Aspirin prevents resistin-induced endothelial dysfunction by modulating AMPK, ROS, and Akt/eNOS signaling. J Vasc Surg. 2012;55(4):1104-15.

29. Silswal N, Singh AK, Aruna B, Mukhopadhyay S, Ghosh S, Ehtesham NZ. Human resistin stimulates the pro-inflammatory cytokines TNF- $\alpha$ and IL-12 in macrophages by NF- $\kappa$ Bdependent pathway. Biochem Biophys Res Commun. 2005;334(4):1092-101.

30. Sarray S, Madan S, Saleh LR, Mahmoud N, Almawi WY. Validity of adiponectin-to-leptin and adiponectin-to-resistin ratios as predictors of polycystic ovary syndrome. Fertil Steril. 2015;104(2):460-6.

31. Singh P, Sridhar M, Rajappa M, Balachander J, Kadhiravan T. Adiponectin-resistin index and its strong association with acute coronary syndrome in South Indian men. Inflammation Research. 2014;63(11):961-8.

32. Chen BH, Song Y, Ding EL, Roberts CK, Manson JE, Rifai N, et al. Circulating levels of resistin and risk of type 2 diabetes in men and women: results from two prospective cohorts. Diabetes Care. 2009;32(2):329-34.

33. Silha JV, Krsek M, Skrha JV, Sucharda P, Nyomba B, Murphy LJ. Plasma resistin, adiponectin and leptin levels in lean and obese subjects: correlations with insulin resistance. Eur J Endocrinol. 2003;149(4):331-5.

34. Wyskida K, Franik G, Wikarek T, Owczarek A, Delroba A, Chudek J, et al. The levels of adipokines in relation to hormonal changes during the menstrual cycle in young, normal-weight women. Endocr Connect. 2017;6(8):892-900.

35. Dafopoulos K, Sourlas D, Kallitsaris A, Pournaras S, Messinis IE. Blood ghrelin, resistin, and adiponectin concentrations during the normal menstrual cycle. Fertil Steril. 2009;92(4):1389-94. 
36. Reseland JE, Mundal HH, Hollung $\mathrm{K}$, Haugen $\mathrm{F}$, Zahid $\mathrm{N}$, Anderssen SA, et al. Cigarette smoking may reduce plasma leptin concentration via catecholamines. Prostaglandins Leukot Essent Fatty Acids. 2005;73(1):43-9.

37. Bokarewa MI, Erlandsson MC, Bjersing J, Dehlin M, Mannerkorpi K. Smoking is associated with reduced leptin and neuropeptide $\mathrm{Y}$ levels and higher pain experience in patients with fibromyalgia. Mediators Inflamm. 2014;2014.

38. Mohamad MM, Mohammad MA, Alomari KS, Karayyem M. Effect of Smoking on Leptin Concentration in Normal Subjects and during Acute Myocardial Infarction. MJIAS. 2009;17(2):7580 .

39. Karaduman M, Oktenli C, Musabak U, Sengul A, Yesilova Z, Cingoz F, et al. Leptin, soluble interleukin- 6 receptor, C-reactive protein and soluble vascular cell adhesion molecule-1 levels in human coronary atherosclerotic plaque. Clin Exp Immunol. 2006;143(3):452-7.

40. Bucholz EM, Beckman AL, Kiefe CI, Krumholz HM. Smoking status and life expectancy after acute myocardial infarction in the elderly. Heart. 2016;102(2):133-9.

41. Haig C, Carrick D, Carberry J, Mangion K, Maznyczka A, Wetherall $\mathrm{K}$, et al. Current smoking and prognosis after acute ST-segment elevation myocardial infarction: new pathophysiological insights. JACC Cardiovasc Imaging. 2019;12(6):993-1003.

42. Shahini N, Michelsen AE, Nilsson PH, Ekholt K, Gullestad L, Broch $\mathrm{K}$, et al. The alternative complement pathway is dysregulated in patients with chronic heart failure. Sci Rep. 2017;7:42532.
43. Ho JE, Lyass A, Courchesne P, Chen G, Liu C, Yin X, et al. Protein biomarkers of cardiovascular disease and mortality in the community. J Am Heart Assoc. 2018;7(14):e008108.

44. Ohtsuki T, Satoh K, Shimizu T, Ikeda S, Kikuchi N, Satoh T, et al. Identification of Adipsin as a Novel Prognostic Biomarker in Patients With Coronary Artery Disease. J Am Heart Assoc. 2019;8(23):e013716.

45. Ermakov S, Azarbal F, Stefanick ML, LaMonte MJ, Li W, Tharp $\mathrm{KM}$, et al. The associations of leptin, adiponectin and resistin with incident atrial fibrillation in women. Heart. 2016;102(17):1354-62.

46. Fukui A, Takahashi N, Nakada C, Masaki T, Kume O, Shinohara $\mathrm{T}$, et al. Role of leptin signaling in the pathogenesis of angiotensin II-mediated atrial fibrosis and fibrillation. Circulation: Arrhythmia and Electrophysiology. 2013;6(2):402-9.

47. Rienstra M, Sun JX, Lubitz SA, Frankel DS, Vasan RS, Levy D, et al. Plasma resistin, adiponectin, and risk of incident atrial fibrillation: the Framingham Offspring Study. Am Heart J. 2012;163(1):119-24. e1.

48. Pascual M, Pascual D, Soria F, Vicente T, Hernandez A, Tebar F, et al. Effects of isolated obesity on systolic and diastolic left ventricular function. Heart. 2003;89(10):1152-6.

49. Finkelhor RS, Moallem M, Bahler RC. Characteristics and impact of obesity on the outpatient echocardiography laboratory. The Am J Cardiol. 2006;97(7):1082-4.

50. Shibata R, Ouchi N, Ohashi $K$, Murohara T. The role of adipokines in cardiovascular disease. J Cardiol. 2017;70(4):32934. 